\section{AB0121 DOES CIGARETTE SMOKING INFLUENCE DISEASE ACTIVITY IN SEROPOSITIVE (ACPA POSITIVE) PATIENTS WITH EARLY RHEUMATOID ARTHRITIS?}

N. Papadopoulos ${ }^{1}$, N. Koletsos ${ }^{2}$, D. Pantelidis ${ }^{1}$, L. Mentesidou ${ }^{3}$

V. Galanopoulou'. 'Papageorgiou General Hospital, Rheumatology Unit, Thessaloniki, Greece; ${ }^{2}$ Papageorgiou General Hospital, Aristotle University of Thessaloniki, ESH Hypertension Excellence Center, 3rd Department of Internal Medicine, Thessaloniki, Greece; ${ }^{3}$ Panagiotis and Aglaia Kyriakou Children's Hospital, First Department of Pediatrics, Athens, Greece

Background: Many studies revealed that cigarette smoking is correlated with more active and severe Rheumatoid Arthritis (RA), mainly through the presence of positive RF or ACPA [1].

Objectives: To investigate the influence of cigarette smoking, in disease activity, in a cohort of Greek patients with ACPA positive early RA.

Methods: From January 2000 until December 2019, 137 patients with seropositive early RA (disease duration $\leq 3$ months), and under the age of 75 years old, were diagnosed and subsequently were followed-up as outpatients in Rheumatology Unit of our hospital. All of them were treated by the same therapeutic protocol, were followed-up every 3 months and had at least one visit during 2019. Demographic, therapeutic, clinical and laboratory parameters were evaluated at the time of diagnosis and in every follow-up. At the end of the study we re-evaluated the above parameters, dividing patients in smokers and non smokers.

Analysis were performed using Student's t-test and non-parametric Mann-Whitney was used to estimate differences between mean values. Pearson's and Spearman's correlations were used, based on the variable's normality of distribution with the "Enter" method was used to identify the statistically significant predicting factors of DAS28/associations with DAS28.

Results: Mean age of our cohort were $57 \pm 10.2$ years and $76.6 \%$ were females. From 137 patients 55 were current smokers.

Univariable analysis at the time of diagnosis revealed, that current smokers presented with more tender $(p<0.001)$, more swollen joints $(p<0.001)$ and higher levels of CRP $(\mathrm{p}<0.001)$. Also current smokers presented with higher disease activity measuring by DAS- $284 v$, than non smokers $(6.2 \pm 1.0 v$ v $5.6 \pm 0.9$ respective, $p<0.001)$. At the end of the study there were not found differences between the two groups.

Multivariable analysis revealed that high DAS-28 was correlated with the intensity of smoking $(p=0.049)$.

Concerning the presence of extra-articular manifestations, smokers were presented more often with rheumatoid nodes $(p=0.026)$.

Conclusion: Current smokers presented with more active disease at the time of diagnosis and have more often rheumatoid nodes. After the establishment of treatment there were no differences in disease activity between smokers and non smokers.

REFERENCES:

[1] Papadopoulos NG et al, Clin Exp Rheum 2005;23:861-66.

Disclosure of Interests: None declared

DOI: 10.1136/annrheumdis-2021-eular.1991

\section{AB0122 THE NORA PROJECT - PREDICTION OF THERAPY RESPONSE IN RHEUMATOID ARTHRITIS}

L. Mathsson-Alm ${ }^{1}$, I. Gehring ${ }^{2}$, M. Poorafshar ${ }^{1}$, J. Rönnelid ${ }^{3}$, J. Askling ${ }^{4}$, E. Haavardsholm ${ }^{5}$, H. Berner Hammer ${ }^{5}$ on behalf of NORA consortium. ${ }^{1}$ Thermo Fisher Scientific, Research and Development, Uppsala, Sweden; ${ }^{2}$ Thermo Fisher Scientific, Research and Development, Freiburg, Germany; ${ }^{3}$ Uppsala University, Department of Immunology, Genetics and Pathology, Uppsala, Sweden: ${ }^{4}$ Karolinska Institutet, Department of Medicine Solna, Stockholm, Sweden; ${ }^{5}$ Diakonhjemmet Hospital, Department of Rheumatology, Oslo, Norway

Background: Personalized medicine in Rheumatoid arthritis (RA) especially regarding therapy response is still in early stages. The Nordic RA (NORA) project is aiming to improve the prediction of therapy outcome by combining established serologic marker with new markers, genetic information and patient-derived data. Objectives: As an initial step in the project the aim was to select clinically characterized patient cohorts and evaluate if changes or patterns in serological markers could predict therapy response and/or disease progress.

Methods: The ARCTIC (Aiming for Remission in rheumatoid arthritis: a randomised trial examining the benefit of ultrasound in a Clinical Tlght Control regimen) study [1] was designed to compare two tight control treatment strategies for early Rheumatoid arthritis and was used as a first cohort. Plasma samples $(n=1622)$ from 224 RA patients from the ARCTIC study were included and taken at baseline and $3,4,6,8,10,12,14,16,20$, and 24 months from trial start, and analyzed for the presence of EliA ${ }^{\mathrm{TM}} \mathrm{RF}(\operatorname{lgM}, \lg A, \lg G)$, anti-CCP $(\operatorname{lgG}, \lg A)$ and anti-RA33 (IgM, IgA, IgG) autoantibodies, as well as Calprotectin using the EliA instrument platform (Phadia AB, Uppsala, Sweden). In addition, a custom-made multiplex chip (Thermo Fisher Scientific, Sweden) [2] was used for measurement of anti-IgG antibodies against RA-specific antigens (citrullinated, acetylated and carbamylated), and established CTD-markers (Connective Tissue Disease), e.g. Ro52/60 and dsDNA. The citrullinated peptides on the multiplex chip were both multiple as well as single citrullinated at different positions within the peptide sequence. Additionally, we included an ELISA to measure antibodies against native human collagen II [3].

Results: The different single assays in the baseline samples varied between 7 $80 \%$ positive test results, e.g. anti-CCP IgG $80 \%$. For some patients we could see changes in levels for anti-CCP, RF and anti-RA33 in the follow up samples, which varied from negative to more than 3-10xULN (Upper Limit of Normal). For anti-CCP $\lg$ we found 9 patients (4\%), who changed from negative to positive (patient 1-5) or from positive to negative (patient 6-8), while patient 9 had a peak at visit $6(=12$ months) and declined afterwards (figure 1). In addition, the above mentioned 9 patients showed clear changes in signal strength for the markers included on the multiplex chip and followed a similar pattern as the anti-CCP IgG signal. Different antibody patterns against single citrullinated peptides were observed and number of ACPA-positive peptides correlated with IgG anti-CCP levels.

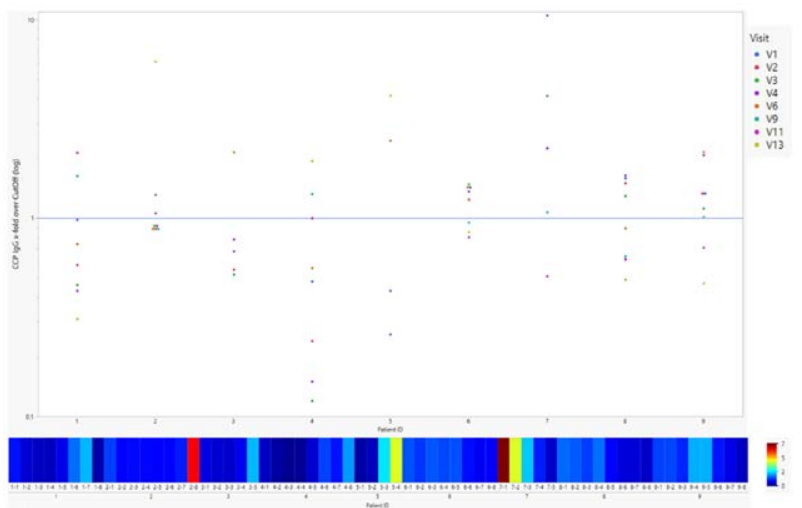

Figure 1. Anti-CCP IgG value normalised to cutoff (blue line) for patient 1 to 9 . The heatmap visualizes the change over time in anti-CCP IgG signal with dark blue showing negative results and orange/red showing results $>5 \times \mathrm{xUN}$.

Anti-Collagen II antibodies (anti-CII) were detected in 15\% of the baseline samples and in most cases declined over time. Two patients showed low baseline anti-CIl levels that increased in the follow up samples. The changes in serological markers and the different reactivity patterns could possibly correlate with clinical outcome and define subgroups of patients with different response to therapy. Results could be repeated in RA patients from the NOR-VEAC [4] cohort. At baseline $73 \%$ of the 106 RA patients had a positive anti-CCP lgG result and 11 patients (10\%) showed a significant change of anti-CCP IgG level over time. Conclusion: Different response patterns and changes in serological antibody levels over the first 24 months after RA diagnosis could possibly reveal subgroups of patients with different prognosis and response to treatment. Further evaluations in additional treatment cohorts and correlation with clinical data are ongoing.

\section{REFERENCES:}

[1] Haavardsholm et al., BMJ 2016;354:i4205.

[2] Hansson et al. Arthritis Research \& Therapy 2012, 14:R201.

[3] Manivel et al Ann Rheum Dis. 2017 Sep;76(9):1529-1536.

[4] Mjaavatten et al., Arthritis Research \& Therapy 2009, 11:R146.

Acknowledgements: The NORA project is a NordForsk funded project.

Disclosure of Interests: Linda Mathsson-Alm Employee of: Employee of Thermo Fisher Scientific, Isabel Gehring Employee of: Employee of Thermo Fisher Scientific, Maryam Poorafshar Employee of: Employee of Thermo Fisher Scientific, Johan Rönnelid: None declared, Johan Askling Grant/research support from: Research grants from Abbvie, Astra-Zeneca, BMS, Eli Lilly, MSD, Pfizer, Roche, Samsung Bioepis, Sanofi, and UCB, mainly in the context of safety monitoring (ARTIS), Espen Haavardsholm: None declared, Hilde Berner Hammer: None declared

DOI: 10.1136/annrheumdis-2021-eular.2024

\section{\begin{tabular}{|l|l}
\hline AB0123 & ANXIETY AND DEPRESSION DURING RHEUMATOID
\end{tabular} ARTHRITIS}

L. Ben Ammar ${ }^{1}$, S. Rekik ${ }^{1}$, S. Boussaid ${ }^{1}$, S. Jemmali ${ }^{1}$, E. Cheour ${ }^{2}$, H. Sahli ${ }^{1}$, M. Elleuch ${ }^{1} .{ }^{1}$ La Rabta Hospital, Rheumatology, Tunis, Tunisia; ${ }^{2}$ La Rabta Hospital, Department of Pain Management, Tunis, Tunisia

Background: Although the management of rheumatoid arthritis (RA) has been booming since the advent of biological treatments, the consequences of this disease remain multiple, both physical and psychological. 
Objectives: The aim of our work was to investigate the factors influencing anxiety and depression in RA.

Methods: This was a cross-sectional study of 49 RA patients. We used the Hospital Anxiety and Depression scale (HAD) to assess anxiety disorders.

Results: We included 24 women and 5 men with an average age of 54.1 years. Sixty-nine percent of patients were unemployed and $81 \%$ had health insurance coverage. RA had been evolving for an average of $11.43 \pm 7.32$ years and the mean time to diagnosis was 2.35 years. It was erosive in $93.8 \%$ of cases. An atlanto-axial dislocation was found in $4.1 \%$ of cases and coxitis in $8.2 \%$ of cases. Eighty-three percent of patients were on cs-DMARDs and $14.2 \%$ were on biotherapy. Most patients had low active disease (53\%) with a mean DAS28CRP score of $2.74 \pm 0.81$. Twelve percent of patients had a probable anxiety and $18 \%$ had a probable depression.

A long delay in diagnosis was associated with a higher risk of anxiety disorders. Working patients had a lower risk of depression while patients from rural areas had a higher risk. The risk of anxiety and depression disorders was associated with increased VAS pain, EGP, and DAS28. In the multivariate study, EGP was the independent risk factor for the development of anxiety and depression disorders. Conclusion: Anxiety and depression disorders are a frequent yet underestimated consequence in RA. Appropriate care in psychiatry is required as soon as the diagnosis is announced.

Disclosure of Interests: None declared

DOI: 10.1136/annrheumdis-2021-eular.2066

\begin{tabular}{|l|l}
\hline AB0124 & STRUCTURAL DAMAGE IN THE FOOT IN \\
& RHEUMATOID ARTHRITIS: DON'T MISS THE OTHER \\
& METATARSOPHALANGEAL JOINTS
\end{tabular}

M. Yasmine ${ }^{1}$, H. Ferjani ${ }^{1}$, D. Kaffel ${ }^{1}$, K. Maatallah ${ }^{1}$, S. Rahmouni ${ }^{1}$, W. Hamdi ${ }^{1}$ ${ }^{1}$ Kassab Institute of Orthopedics, Rheumatology, Tunis, Tunisia

Background: The foot involvement in rheumatoid arthritis (RA) affects the functionality and the quality of life in patients. Despite this, the clinicians do not give enough care to the foot in RA patients, especially if asymptomatic, resulting in joint damage, deformity, and disability. The distribution of erosions of the other MTP joints (excluding the 5th) has not previously been studied.

Objectives: This study aimed to investigate the distribution of erosions in MTP joints and their clinical implications.

Methods: We conducted a retrospective study including patients with RA according to the American college of rheumatology/ the European league against rheumatism classification criteria. Sociodemographic data, as well as disease activity related characteristics, were recorded.

Posterior-anterior radiographs of the hands and feet of each patient were assessed for erosions.

All patients were assessed by Ultrasonography (US) of the hands. US erosions were scored 0-3 according to Szkudlarek [1]. We divided patients into two groups (G1 without MTPs erosions and G2 with MTPs erosions).

Results: We enrolled forty-two females and eleven males in our studies. The mean age was 58.6 years \pm 12.7 [23-77], and the mean disease duration was 8.4 years [1-47]. Rheumatoid factor or cyclic citrullinated peptide antibodies (Anti-CCP) were positive in $62.3 \%$ of cases. The mean DAS28ESR score was $5.1 \pm 1.16$ [2.5-7.7]. Half of the patients had the active disease $(52.8 \%)$. Hand erosions evaluated with plain radiographs and the US were found in $43.1 \%$ and $50.9 \%$ of cases, respectively. The distribution of foot erosions (15.1\%) was at follows: 5th right MTP $(7.5 \%)$, the 5 th right IPP (2\%), the 1st left MTP (2\%), 3th left MTP $(3.8 \%)$, 4th left MTP $(5.7 \%)$ and the 5th left MTP $(9.4 \%)$. Erosions on MTPs with the exclusion of the 5th MTP were present in $9.4 \%$ of cases. The presence of MTPs erosion was more frequent in males $(p=0.01)$ but was not associated with age $(p=0.6)$ or disease duration $(p=0.2)$. Seropositivity was similar between the two groups $(p=0.06)$. Similarly, the inflammatory markers (ESR and CRP), as well as DAS28 ESR, did not differ between the two groups ( $p>0.05)$. MTPs erosion was not associated with the presence of hand erosions on a plain radiograph $(p=0.445)$. However, MTPs erosion was significantly more frequent in patients with less erosive hands-on US $(p=0.034)$.

Conclusion: Our study showed that screening of other MTPs (excluding the 5th) is mandatory in RA diagnosis. Interestingly, in our result, MTPs erosion is more frequent in males with less erosive hands.

Disclosure of Interests: None declared

DOI: 10.1136/annrheumdis-2021-eular.2227

\section{AB0125 1 CLINICAL CHARACTERISTICS OF RHEUMATOID ARTHRITIS PATIENTS WITH IGG4-RELATED SYNOVITIS}

L. F. Chen ${ }^{1}$, X. Zhang ${ }^{1}$, C. Chen ${ }^{1}$, J. D. Ma ${ }^{1}$, Y. Mo ${ }^{1}$, J. Lin ${ }^{1}$, Y. Y. Zou ${ }^{1}$, D. H. Zheng ${ }^{1}$, L. Dai ${ }^{1}$. 'Sun Yat-Sen Memorial Hospital, Sun Yat-Sen University, Rheumatology, Guangzhou, China

Background: Elevated serum IgG4 (slgG4) and IgG4+ plasma cell tissue infiltration are outstanding features of IgG4-related disease (IgG4-RD). However, elevated
IgG4 is not specific for IgG4-RD. Our previous study reported elevated slgG4 in 46\% of rheumatoid arthritis (RA) patients (Mediators Inflamm 2014). Whether synovium from RA patients show similar characteristics of IgG4-RD and how about the clinical characteristics of RA patients with IgG4-related synovitis have not been reported yet. Objectives: To explore the serum and synovial IgG4 level and their correlation with disease indicators in RA.

Methods: Active RA patients who underwent needle synovial biopsy with qualified synovium tissue were recruited. Demographic and clinical data were collected simultaneously. Synovium tissue were stained with H\&E for Krenn synovitis score and immunohistochemistry for positive cell densities of CD20, CD38, IgG and IgG4. Serum IgG4 level was detected by immunonephelometry.

Results: Among 96 RA patients recruited, 74 (77.1\%) were female, the median age was 55.0 (46.0 61.0) years, disease duration was $42.0(12.0 \sim 120.0)$ months and SDAI was $31.2(22.1 \sim 42.8)$

The median slgG4 was 1.38 (0.86 2.42) $\mathrm{g} / \mathrm{L}$ and $49(51.0 \%)$ patients had elevated slgG4. Compared with those with normal slgG4, RA patients with elevated slgG4 had significantly higher levels of PrGA [7 (5 8) vs. 6 (4 7)], ESR [90 (64 116) mm/h vs. $61(38 \sim 75) \mathrm{mm} / \mathrm{h}]$, CRP $[46.20(17.20 \sim 74.20) \mathrm{mg} / \mathrm{L}$ vs. $18.90(9.46 \sim 49.20)$ $\mathrm{mg} / \mathrm{L}]$, DAS28-ESR $[6.3(5.6 \sim 7.4)$ vs. 5.7 (4.7 6.4)], SDAl [34.2 (25.3 48.8) vs 27.8 (18.9 35.9)] and HAQ-DI [1.70 (0.61 2.28) vs. $0.88(0.40 \sim 1.75)$, all $P<0.05]$ Meanwhile, they also showed significantly higher synovial counts of CD38+ plasma cells [1240(559 2290) $/ \mathrm{mm}^{2}$ vs. 1020(354 1777) $\left./ \mathrm{mm}^{2}\right]$, IgG4+ plasma cells [106 (39 249) $/ \mathrm{mm}^{2}$ vs. $68(3 \sim 123) / \mathrm{mm}^{2}$ ], and higher ratio of IgG4+/lgG+ plasma cells [26.3 (15.5 38.0) \% vs. $15.2(0.9 \sim 24.7) \%$, all $P<0.05$ ]

The median IgG4+ plasma cells count was $83(10 \sim 192) / \mathrm{mm}^{2}$ and median ratio of IgG4+/lgG+ plasma cells was $19.1(8.4 \sim 31.5) \%$. Both of them correlated positively with ESR, CRP and slgG4 $(r=0.216 \sim 0.394$, all $P<0.05)$. There were 46 $(47.9 \%)$ patients with IgG4+ plasma cells $>10 / \mathrm{HPF}$, who had significant higher ESR [86 (50 109) mm/h vs. 65 (40 84) mm/h] and CRP [43.35 (16.93 77.85) $\mathrm{mg} / \mathrm{L}$ vs. $26.15(9.54 \sim 52.53) \mathrm{mg} / \mathrm{L}$, both $P<0.05]$ than those with IgG4+ plasma cells $\leq 10 / \mathrm{HPF}$. There were $13(13.5 \%)$ patients with the ratio of $\operatorname{lgG} 4+/ \lg \mathrm{H}_{+}$ plasma cells $>40 \%$, and $11(11.5 \%)$ patients with both IgG4+ plasma cells $>10 /$ HPF and IgG4+/lgG+ plasma cells ratio $>40 \%$ (IgG4-related synovitis). RA patients with IgG4-related synovitis had significant higher ESR than the others [106 (53 125) mm/h vs. 69 (41 91) $\mathrm{mm} / \mathrm{h}, P<0.05$ ]

There were 10 (10.4\%) patients showing elevated slgG4 and IgG4-related synovitis. Four patients completed 1-year follow-up and all of them achieved remission at $6^{\text {th }}$ month (SDAl $\leq 3.3$, Figure 1). Only one patient had radiographic progression at $12^{\text {th }}$ month.
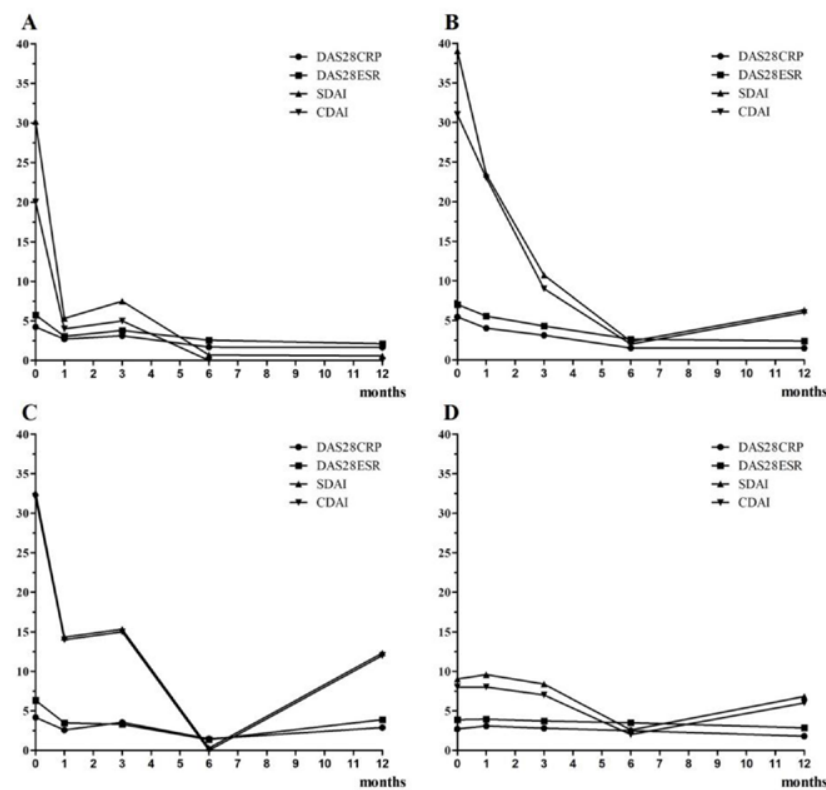

Figure 1. Dynamic disease activity of 4 RA patients with elevated slgG4 and IgG4-related synovitis during 1-year follow-up.

Conclusion: IgG4-related synovitis can be found in RA patients. Their clinical significance in disease characteristics and outcomes are worth further study. Acknowledgements: This work was supported by National Natural Science Foundation of China (no. 81971527, 81801606 and 81801605), Guangdong Natural Science Foundation (no. 2018A030313541 and 2018A030313690) Guangdong Medical Scientific Research Foundation (no. A2018062), Guangdong Basic and Applied Basic Research Foundation (no. 2019A1515011928 and 2020A1515110061), and Science and Technology Program of Guangzhou (no. 201904010088) 